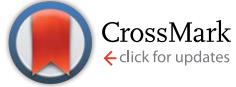

Cite this: RSC Adv., 2015, 5, 14697

Received 2nd December 2014 Accepted 16th January 2015

DOI: 10.1039/c4ra15635h

www.rsc.org/advances

\section{Unsymmetric vesicles with a different design on each side for near-infrared fluorescence imaging of tumor tissues $\uparrow$}

\begin{abstract}
Akihiro Uesaka, ${ }^{a}$ Isao Hara, ${ }^{\text {b }}$ Tomoya Imai, ${ }^{c}$ Junji Sugiyama ${ }^{c}$ and Shunsaku Kimura*a
Spherical vesicular assemblies with unsymmetric membranes are prepared from $A_{3} B$-type and $A B^{\prime}$-type peptides with different helix senses in the hydrophobic $B$ and $B^{\prime}$ blocks under the control of three associated factors: stereo-complex formation, dipole-dipole interactions, and steric effects. [(Sar $\left.)_{26}\right]_{3}-b$ ( $L$-Leu-Aib) $6\left(A_{3} B L\right),(S a r)_{27}-b$-(L-Leu-Aib) $6(A B L),(S a r)_{28}-b$-(D-Leu-Aib) 6 (ABD), lipoic-acid terminated $A B L$ (lipoABL), and lipoic-acid terminated $A B D$ (lipoABD) are mixed while keeping equimolar ratios of the total amounts of the right- and the left-handed helices. When $\mathrm{A}_{3} \mathrm{BL}$ exceeds more than $60 \%$ of all the righthanded helices of the mixture, the unsymmetric vesicles are formed, as indicated by the selective adsorption of gold nanoparticles on the vesicle surface observed by TEM. The unsymmetric vesicles are also prepared using ICGABL and ICGABD with a near-infrared fluorophore, indocyanine green (ICG), at the terminals of $A B L$ and $A B D$. The unsymmetric vesicle formation is verified by fluorescence quenching with the addition of In ions to the solution. When these vesicles are injected into tumor-bearing mice, the vesicles are effectively accumulated into tumor tissues via the EPR effect. The unsymmetric vesicle containing ICG at the inward surface induces less IgM production than that with ICG at the outward surface. More stealth vesicles in the blood stream are therefore obtainable by concealing the imaging fluorophores in the vesicle.
\end{abstract}

\section{Introduction}

Amphiphilic molecules self-assemble into molecular assemblies in water via various intermolecular interactions such as hydrophobic and electrostatic interactions. ${ }^{1-5}$ Their morphologies depend heavily on the chemical structure of the components, and suitable molecular designs have been proposed to generate molecular assemblies with various morphologies and sizes. ${ }^{6-8}$ In addition, molecular assemblies have been applied for broad fields ranging from nanodevices to biomaterials. ${ }^{9-14}$

Among them, vesicles are one of the most frequently appearing nanocarriers for tumor-targeted drug delivery and molecular imaging. ${ }^{15-20}$ In tumor tissues, the vascular walls have submicron holes because of rapid angiogenesis; therefore, nanocarriers can permeate into tumors through the vascular wall. Furthermore, the lymph system around tumors grows so slowly that nanocarriers are usually retained in the tumor. This

${ }^{a}$ Department of Material Chemistry, Graduate School of Engineering, Kyoto University, Kyoto-Daigaku-Katsura, Nishikyo-ku, Kyoto, 615-8510, Japan. E-mail: shun@scl. kyoto-u.ac.jp; Fax: +81-75-383-2401; Tel: +81-75-383-2400

${ }^{b}$ Technology Research Laboratory, Shimadzu Corporation, 3-9-4 Hikari-dai, Seika-cho, Soraku-gun, Kyoto 619-0237, Japan

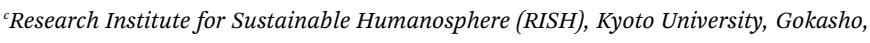
Uji, Kyoto 611-0011, Japan

$\dagger$ Electronic supplementary information (ESI) available. See DOI: $10.1039 / \mathrm{c} 4 \mathrm{ra} 15635 \mathrm{~h}$ effect is called the enhanced permeability and retention (EPR) effect. ${ }^{21}$ In this regard, the control of the nanocarrier size is essential to allow them to be passively accumulated into tumor tissues. ${ }^{18,22,23}$ Various vesicles of liposomes, polymersomes, and peptosomes, which are prepared from lipids, ${ }^{24,25}$ polymers, ${ }^{26,27}$ and peptides, ${ }^{18,28}$ respectively, have been studied. One challenge of vesicular molecular assemblies is to prepare unsymmetric vesicles with different components between the outer and the inner surfaces that mimic cell membranes. For example, Fuhrhop reported that unsymmetric bolaamphiphiles with small and large head groups self-assemble into unsymmetric monolayer vesicles via steric effects. ${ }^{29,30}$ Furthermore, Xiao and Pautot reported that reverse micelles were transformed into lipid monolayers at the oil/water interface to form unsymmetric vesicles. ${ }^{31,32}$ However, it is not so easy to prepare unsymmetric membranes under precise control of the distribution of the components. In addition, these methods do not seem to be applicable to the unsymmetric self-assembly of amphiphilic polymers due to the disordered packing and/or molecular entanglement of the hydrophobic blocks.

We previously reported that left-handed helical peptides with one hydrophilic chain (AB-type) and right-handed helical peptides with three hydrophilic chains $\left(\mathrm{A}_{3} \mathrm{~B}^{\prime}\right.$-type) co-assemble into unsymmetric peptosomes via stereo-complex formation, dipole-dipole interactions, and steric effects. ${ }^{33}$ Stereo-complex formation and dipole-dipole interactions can result in 


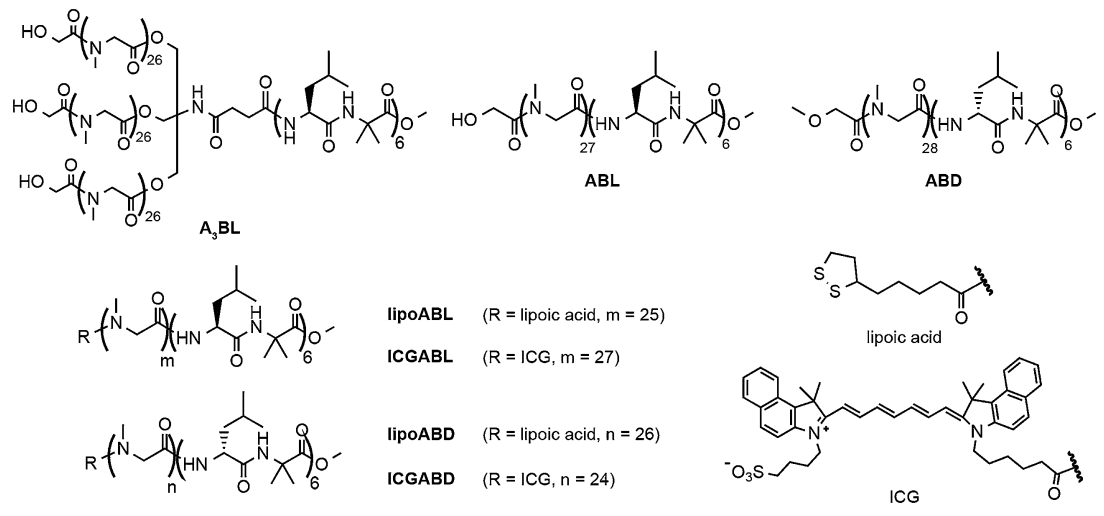

Fig. 1 Chemical structures of amphiphilic block polypeptides $A_{3} B L, A B L, A B D$, lipoABL, lipoABD, ICGABL, and ICGABD.

regularly interdigitated helix packing to generate unsymmetric planar sheets. The planar sheets grew into unsymmetric vesicles due to the steric effect of the bulky hydrophilic $A_{3}$ moieties of the $\mathrm{A}_{3} \mathrm{~B}$-type peptides extruding to the outer surface. Furthermore, the selective surface modification at the outer or inner surface can be easily performed by choosing the right-handed or left-handed helix of the functionalised helical peptides.

In this paper, we study a necessary proportion of the $\mathrm{A}_{3} \mathrm{~B}$ type helix, $\left[(\mathrm{Sar})_{26}\right]_{3}-b$-(L-Leu-Aib) ${ }_{6}\left(\mathbf{A}_{3} \mathbf{B L}\right)$, for the generation of unsymmetric vesicles in mixtures of the $\mathrm{A}_{3} \mathrm{~B}$-type righthanded helical peptide $\left(\mathbf{A}_{3} \mathbf{B L}\right)$, AB-type right-handed helical peptide, $\quad(\mathrm{Sar})_{27}-b$-(L-Leu-Aib) 6 (ABL), AB-type left-handed helical peptide, $(\mathrm{Sar})_{28}-b$-(D-Leu-Aib) ${ }_{6}(\mathbf{A B D})$, and two AB-type helical peptides with lipoic acid at the termini of the hydrophilic chains, lipoABL and lipoABD (Fig. 1). Lipoic acid at the terminal of the poly(sarcosine) chain has been shown to behave as a hydrophilic group in these molecular assemblies. ${ }^{33,34}$ Unsymmetric vesicles about the location of near-infrared fluorescent (NIRF) probe are also prepared here using the novel functional AB-type helical peptides modified with indocyanine green (ICG), ICGABL and ICGABD (Fig. 1). Using these two types of unsymmetric vesicles, one with ICG groups on the outer surface and the other with ICG groups on the inner surface, we examine the in vivo distributions of these vesicles and the IgM productions in tumor-bearing mice to obtain information of the effect of surface modification on the pharmacokinetics of the vesicles.

\section{Experimental section}

\subsection{Materials}

The amphiphilic peptides $\mathbf{A}_{\mathbf{3}} \mathbf{B L}, \mathbf{A B L}, \mathbf{A B D}$, lipoABL and lipoABD were obtained as reported previously. ${ }^{33,34}$ ICGABL and ICGABD were synthesised according to Scheme S1 (ESI $\dagger$ ). The synthetic details are described in the ESI. $\uparrow$ The near-infrared fluorescence compound, ICG-Sulfo-OSu, was purchased from Dojin Laboratory Ltd. Au nanoparticles (AuNPs) were purchased from BB international (U.K.) with an average diameter of $10 \mathrm{~nm}$. All reagents were purchased commercially and used as received unless otherwise noted.

\subsection{Preparation of molecular assembly}

A solution of polypeptides in ethanol $\left(0.05 \mathrm{mg} \mu \mathrm{L}^{-1}, 5.0 \mu \mathrm{L}\right)$ was injected into milliQ water under stirring at $4{ }^{\circ} \mathrm{C}$. In the case of $\mathbf{A}_{\mathbf{3}} \mathbf{B L}$, methanol was used instead of ethanol. After $30 \mathrm{~min}$, the dispersion was heated by an aluminium heat source if necessary, followed by cooling at room temperature for measurements.

\subsection{Transmission Electron Microscopy (TEM)}

TEM images were collected using a JEOL JEM-2000EXII at an accelerating voltage of $100 \mathrm{kV}$. Peptide aqueous solutions were applied on a carbon-coated $\mathrm{Cu}$ grid, and the samples were negatively stained with $2 \%$ uranyl acetate followed by suction of the excess fluid with a filter paper.

\subsection{Frozen-hydrated/cryogenic-TEM (Cryo-TEM)}

The peptide dispersions were quickly frozen in liquid ethane, which was cooled with liquid nitrogen. The samples were examined at an accelerating voltage of $100 \mathrm{kV}$ at the temperature of liquid nitrogen.

\subsection{Dynamic Light Scattering (DLS)}

The DLS measurements were taken using a DLS-8000KS (Photal Otsuka Electronics) with a He-Ne laser at $25{ }^{\circ} \mathrm{C}$. Before the DLS measurements, each prepared sample was filtered using a $0.20 \mu \mathrm{m}$ PVDF (polyvinylidene fluoride) syringe filter (GE Healthcare UK limited).

\subsection{The adhesion of AuNPs to vesicles}

Preliminarily, peptides with lipoic acid were mixed with the solution of amphiphilic peptides, and the molecular assemblies were prepared from the solutions according to the previously described procedure. ${ }^{33}$ The peptide aqueous solutions were applied on a carbon-coated $\mathrm{Cu}$ grid, and AuNPs with diameters of $10 \mathrm{~nm}$ were then added on the grid to reduce the non-specific association of AuNPs with vesicles. The lipoic acids on the molecular assemblies can easily react with AuNPs on the $\mathrm{Cu}$ grid. ${ }^{33,34}$ After washing the excess AuNPs with dispersion media, 
the samples were negatively stained with $2 \%$ uranyl acetate followed by suction of the excess fluid with a filter paper.

\subsection{Fluorescent analysis}

The fluorescent spectra of the peptide dispersions were obtained using a JASCO FP-6600 spectrofluorometer at an optional temperature with a transmission cell with an optical path length of $1 \mathrm{~cm}$. Before measurements, vesicle suspensions were diluted to $0.05 \mathrm{mg} \mathrm{mL}^{-1}$ (in a total volume of $2.0 \mathrm{~mL}$ ). The excitation wavelength of ICG is $755 \mathrm{~nm}$.

\subsection{In vivo NIRF-imaging of vesicles with ICG}

SUIT2/pEF/LUC cells $\left(5 \times 10^{5}\right.$ cells $)$ were dissolved in PBS $(20 \mu \mathrm{L})$ and subcutaneously inoculated into the right femoral region of seven-week-old BALB/c nu/nu mice ( $n=3$ per group). Vesicles containing ICG were prepared in milliQ water, and $\mathrm{NaCl}$ was added into the suspensions to adjust to physiological conditions ( $0.9 \mathrm{wt} \% \mathrm{NaCl}$ aq.). The vesicle suspensions $\left(5 \mathrm{mg} \mathrm{kg}^{-1}, 100 \mu \mathrm{L}\right)$ were injected into the mice via the tail vein one week after the tumor transplantation. The amount of

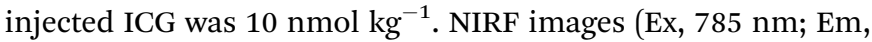
$845 \mathrm{~nm}$ ) were taken at $15 \mathrm{~min}, 1 \mathrm{~h}, 3 \mathrm{~h}, 6 \mathrm{~h}, 9 \mathrm{~h}$, and $24 \mathrm{~h}$ after the dose. During the imaging process, the mice were held on the imaging stage under anesthetised conditions with $2.5 \%$ of isoflurane gas in air flow $\left(1.5 \mathrm{~L} \mathrm{~min}^{-1}\right)$. The pseudo images were constructed from the photon counts.

\subsection{IgM assay}

For ELISA, vesicles $\left(5 \mathrm{mg} \mathrm{kg}^{-1}, 100 \mu \mathrm{L}\right)$ were injected to the $\mathrm{BALB} / \mathrm{c} \mathrm{nu} / \mathrm{nu}$ mice, which were purchased from Japan SLC, Inc. (Japan; $n=3$ per group). Mouse blood was collected in $1.5 \mathrm{~mL}$ tubes from the inferior vena cava under anaesthesia conditions at one week after administration. The serum was kept overnight at $4{ }^{\circ} \mathrm{C}$. The next day, the serum was obtained by centrifugation (10 min, $3000 \mathrm{rpm}$ ) and saved at $-40{ }^{\circ} \mathrm{C}$.

Lactosomes prepared from $\left[\text { poly }(\mathrm{Sar})_{50}\right]_{3}-b$-poly(L-lactic acid $)_{30}$ in $50 \mu \mathrm{L}$ acetonitrile were added to 96-well plates $(0.5 \mu \mathrm{g}$ per well) and air dried completely for one day. Then, $150 \mu \mathrm{L}$ of blocking buffer $[2 \% \mathrm{BSA} /$ phosphate-buffered saline (PBS)] was added and incubated for $2 \mathrm{~h}$. The wells were washed four times with washing buffer (PBS-T: 0.05\% tween 20 in PBS). The serially diluted sera were added to the wells and incubated for $2 \mathrm{~h}$. After incubation, the wells were washed four times with PBS-T. Peroxidase-conjugated goat-anti-mouse IgM in $0.1 \%$ BSA/PBS (50 $\mu \mathrm{L}$, Southern Biotech, USA) was added as the secondary antibody. After the solution was incubated for $2 \mathrm{~h}$, the wells were again washed four times with PBS-T. $o$-Phenylenediamine (0.5 mg mL ${ }^{-1}$, Sigma, St Louis, MO) dissolved in $0.0003 \% \mathrm{H}_{2} \mathrm{O}_{2}-$ $0.1 \mathrm{M}$ citrate phosphate buffer ( $\mathrm{pH}$ 5.0) was added to the wells. At $10 \mathrm{~min}$ after the $o$-phenylenediamine addition, the OD was determined at $490 \mathrm{~nm}$ with $620 \mathrm{~nm}$ as a reference.

\subsection{Statistical analysis}

All results are expressed as mean \pm SD. Differences between groups in amount of AuNPs attached on vesicles, IgM production and NIRF-imaging studies were assessed by the $t$ test for independent samples. $P$-values $<0.05$ and $<0.01$ were considered statistically significant and designated by * and **, respectively.

\subsection{Ethics}

All of our in vivo animal experiments were approved by the animal research committee of Kyoto University. The animals were treated humanely.

\section{Results and discussions}

\subsection{Preparation of unsymmetric vesicles}

$\mathbf{A}_{3} \mathbf{B L}$ and $\mathbf{A B D}$ were co-assembled into planar sheets with lengths of $c a .150 \mathrm{~nm}$ in milliQ water (Fig. 2a), and these sheets were transformed into vesicles with diameters of $c a .90 \mathrm{~nm}$ upon heating at $90{ }^{\circ} \mathrm{C}$ for $1 \mathrm{~h}$ (Fig. 2b). DLS measurements showed that the vesicle diameter was $99.6 \mathrm{~nm}$ with a narrow size distribution (PDI $=0.052)$. These results suggest that the stereocomplex formation contributes to the formation of the planar sheet morphology, and the subsequent heat treatment triggers vesicle formation so as to reduce the hydrophobic edges in water, as reported previously. ${ }^{33-35}$

To study the unsymmetry of the vesicle, lipoABL and lipOABD, which can bind AuNPs, were incorporated into the vesicles prepared from $\mathbf{A}_{3} \mathbf{B L}$ and $\mathbf{A B D}$, respectively. ${ }^{33}$ In a previous paper, we demonstrated that lipoic acid at the hydrophilic chain terminal behaves as a hydrophilic moiety. ${ }^{33,34}$ If the lipoic acids of amphiphilic peptides are oriented towards the inside of vesicles, AuNPs should not adhere to the vesicles, which can be visualised by TEM to provide information on the peptide orientation in the membrane. AuNPs adhered clearly to the vesicles prepared from $\mathbf{A}_{3} \mathbf{B L}+\mathbf{A B D}+\operatorname{lipoABL}(0.4 / 0.5 / 0.1, \mathrm{~mol} /$ $\mathrm{mol} / \mathrm{mol}$; Fig. 3a and S2a of ESI $\dagger$ ), whereas few AuNPs adhered to the vesicles prepared from $\mathbf{A}_{3} \mathbf{B L}+\mathbf{A B D}+\operatorname{lipoABD}(0.5 / 0.4 / 0.1$, $\mathrm{mol} / \mathrm{mol} / \mathrm{mol}$; Fig. $3 \mathrm{~b}$ and S2b of ESI†). The TEM images show the collapsed vesicles due to the vacuum condition of the TEM observation, but the morphology was confirmed to be spherical by cryo-TEM observation (Fig. 5). These results indicate that the unsymmetric vesicles were formed from the ternary mixtures, and the functionalised peptides with lipoic acid were integrated into the membrane under control of the molecular orientation according to the right- or left-handed helical sense of the functionalised peptides.
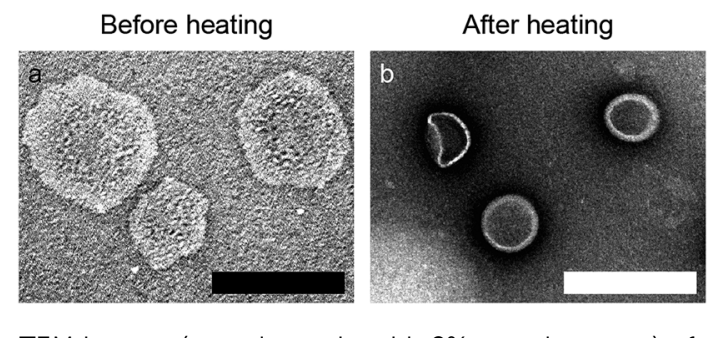

Fig. 2 TEM images (negative stain with $2 \%$ uranyl acetate) of molecular assemblies prepared from $\mathrm{A}_{3} \mathrm{BL}$ and $\mathrm{ABD}$ in milliQ water (a) before and (b) after heat treatment at $90{ }^{\circ} \mathrm{C}$ for $1 \mathrm{~h}$. Scale bars are $200 \mathrm{~nm}$. 
$10 \mathrm{~mol} \%$ lipoABL
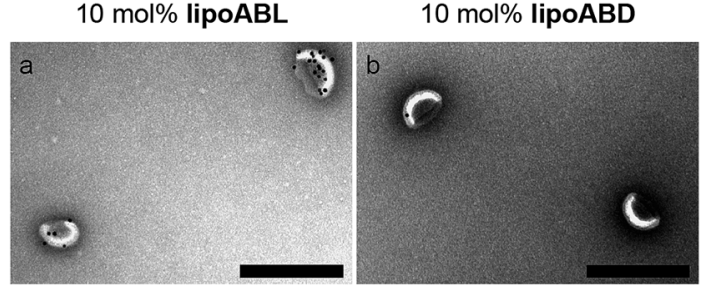

Fig. 3 TEM images (negative stain with 2\% uranyl acetate) of vesicles prepared from (a) $A_{3} B L+A B D+$ lipo $A B L$ and (b) $A_{3} B L+A B D+$ lipoABD in milliQ water upon heat treatment at $90{ }^{\circ} \mathrm{C}$ for $1 \mathrm{~h}$. An aliquot of a $10 \mathrm{~nm}$ AuNP suspension was added to the vesicles on the TEM grid. Scale bars are $200 \mathrm{~nm}$.

\subsection{The effect of $\mathrm{A}_{3} \mathrm{BL}$ proportion on unsymmetric membranes}

To evaluate the proportion of $\mathbf{A}_{\mathbf{3}} \mathbf{B L}$ in all the right-handed helices necessary to achieve the unsymmetric vesicles by steric effects, the compositions of $\mathbf{A B L}$ and $\mathbf{A}_{\mathbf{3}} \mathbf{B L}$ were varied (Fig. 4). As described above, several AuNPs adhered to the vesicles of $\mathbf{A}_{3} \mathbf{B L}+\mathbf{A B D}+$ lipoABL $(0.4 / 0.5 / 0.1, \mathrm{~mol} / \mathrm{mol} / \mathrm{mol})$, but few AuNPs adhered to those of $\mathbf{A}_{\mathbf{3}} \mathbf{B L}+\mathbf{A B D}+$ lipoABD (0.5/0.4/0.1, $\mathrm{mol} / \mathrm{mol} / \mathrm{mol})$. The extent of the latter AuNP adherence was similar to that to the vesicles without the lipoic acid-modified peptides (Fig. S3e of ESI $\dagger$ ), suggesting the nonspecific adherence of AuNPs in these cases. The quaternary mixture vesicle of $\mathbf{A}_{3} \mathbf{B L}, \mathbf{A B L}, \mathbf{A B D}$, and lipoABL (0.30/0.10/0.50/ 0.10 , vesicle $\mathrm{c}$ in Fig. 4), which corresponds to a $60 \%$ proportion of the $\mathrm{A}_{3} \mathrm{~B}$-type peptide in all the right-handed helices, clearly attached to AuNPs (Fig. S3a of ESI $\dagger$ ). On the other hand, when lipoABL was replaced by lipoABD with the same $60 \%$ proportion of the $\mathrm{A}_{3} \mathrm{~B}$-type peptide in all the right-handed helices, the quaternary mixture vesicle of $\mathbf{A}_{\mathbf{3}} \mathbf{B L}, \mathbf{A B L}, \mathbf{A B D}$,

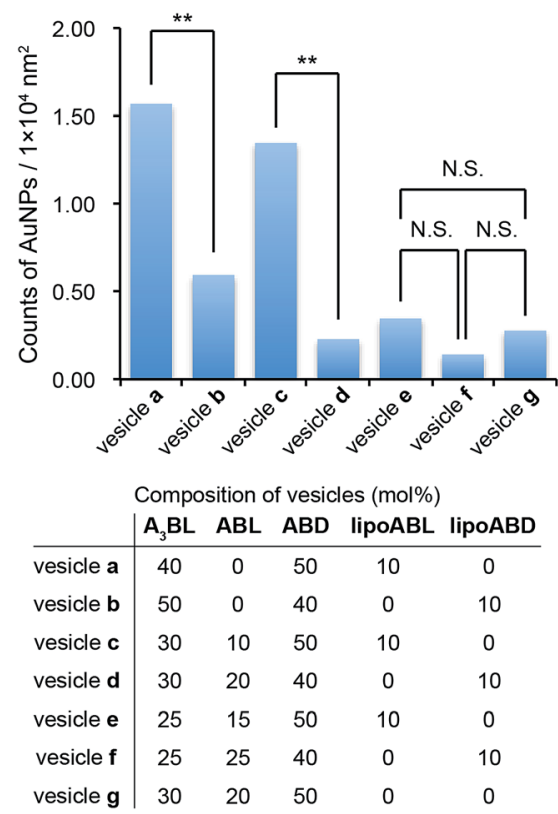

Fig. 4 The number of AuNPs per unit area $\left(100 \times 100 \mathrm{~nm}^{2}\right)$ of vesicle surface. The $p$-value: ${ }^{* *} p<0.01$. and lipoABD $(0.30 / 0.20 / 0.40 / 0.10$, vesicle $d$ in Fig. 4$)$ attached to significantly fewer AuNPs (Fig. S3b of ESI $\dagger$ ). It is therefore indicated that the orientation of the peptides should be maintained in the unsymmetric way even in the vesicles containing a $60 \%$ proportion of the $\mathrm{A}_{3} \mathrm{~B}$-type peptide. The numbers of AuNPs adhered per unit surface area of the vesicles in the TEM images were counted, and the data were analysed statistically (Fig. 4). The selective adherence of AuNPs to vesicles a, b, $c$, and $d$ was confirmed statistically by a $p$-value of less than 0.01. It is therefore concluded that all the right-handed helices in these vesicles should orient towards the outside of the vesicle.

However, when the proportion of the $\mathrm{A}_{3} \mathrm{~B}$-type peptide decreased to $50 \%$ in all the right-handed helices (vesicles e and $\mathrm{f}$ in Fig. 4), the extent of AuNP adherence was similar between these vesicles (Fig. S3c and d of ESI $\dagger$ ). These results suggest that the molecular direction of helices in the membrane cannot be controlled when the vesicles contain less than 50\% $\mathrm{A}_{3} \mathrm{~B}$-type peptide in all the right-handed helices.

The DLS measurements revealed that the vesicle diameter increased by increasing the proportion of $\mathbf{A B L}$ or, in other words, by decreasing the proportion of $\mathbf{A}_{3} \mathbf{B L}$ (Fig. S4 of $\mathrm{ESI}_{\dagger} \dagger$ ). This result suggests that substituting AB-type peptides for bulky $\mathrm{A}_{3} \mathrm{~B}$-type peptides decreases the curvature of the vesicles. Furthermore, the diameter increases sharply when the proportion of ABL in all the right-handed helices is more than $50 \%$. This increase also supports the interpretation that bulky $\mathrm{A}_{3} \mathrm{~B}$ type peptides start to orient towards the inside of some vesicles when the proportion of ABL is more than 50\%. From these results, it is concluded that unsymmetric vesicles can be prepared in the presence of $\mathbf{A}_{\mathbf{3}} \mathbf{B L}$ at a proportion of greater than $60 \%$ in all the right-handed helices, and the functionalised unsymmetric vesicles are applicable for some applications that require unsymmetric membranes.

\subsection{The application of unsymmetrical vesicles to NIRF imaging}

The unsymmetric vesicles were examined as nanocarriers for the NIRF imaging of solid tumor. The ternary mixtures of $\mathbf{A}_{3} \mathbf{B L}+\mathbf{A B D}+$ ICGABL $(0.48 / 0.5 / 0.02, \mathrm{~mol} / \mathrm{mol} / \mathrm{mol})$ and $\mathbf{A}_{3} \mathbf{B L}+$ ABD + ICGABD $(0.5 / 0.48 / 0.02, \mathrm{~mol} / \mathrm{mol} / \mathrm{mol})$ were selfassembled into homogeneous vesicles in milliQ water (Fig. 5 and S5 of ESI $\dagger$ ); the corresponding vesicle diameters determined by DLS were $111.7 \mathrm{~nm}$ and $111.5 \mathrm{~nm}$, respectively, which are in agreement with the previously described unsymmetric vesicles. The cryo-TEM images confirm the formation of vesicles, even in the presence of ICG-peptides in these compositions (Fig. 5).

To confirm that vesicles containing ICG also have unsymmetric membranes, aqueous $\mathrm{InCl}_{3}$ was added to the vesicle suspension as a quencher because the hydrophilic poly(sarcosine) chains of vesicles have been suggested to adsorb $\mathrm{In}^{3+}$ ions to effectively quench ICG fluorescence via the heavyatom effect. When $10 \mu \mathrm{M}$ aqueous $\mathrm{InCl}_{3}$ was added to vesicles containing ICG-peptides, In $^{3+}$ ions more effectively quenched the fluorescence from the vesicle containing ICGABL than from 

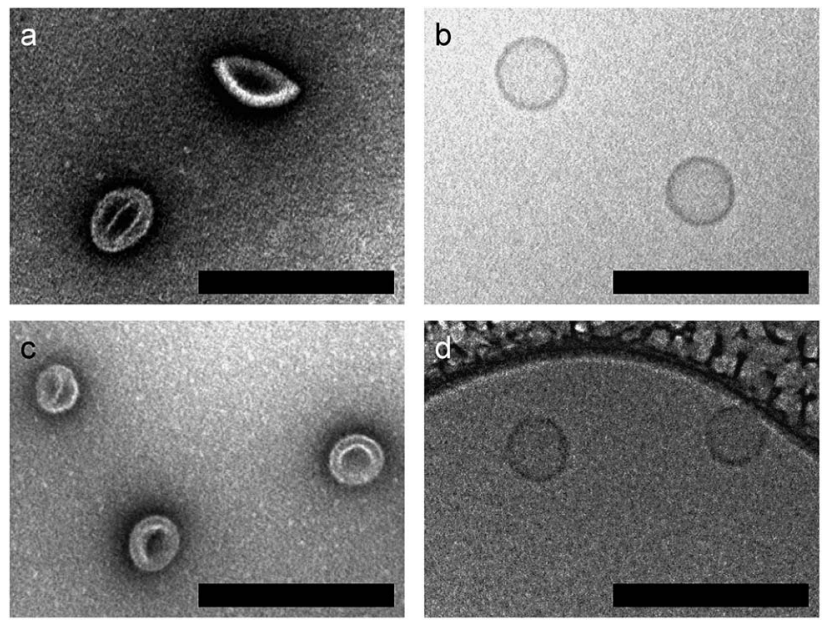

Fig. 5 TEM images (negative stain with $2 \%$ uranyl acetate) of vesicles prepared from (a) $A_{3} B L+A B D+I C G A B L$ and (c) $A_{3} B L+A B D+I C G A B D$ in milliQ water upon heat treatment at $90{ }^{\circ} \mathrm{C}$ for $1 \mathrm{~h}$. Cryo-TEM images of vesicles prepared from (b) $A_{3} B L+A B D+I C G A B L$ and (d) $A_{3} B L+$ ABD + ICGABD. Scale bars are $200 \mathrm{~nm}$.

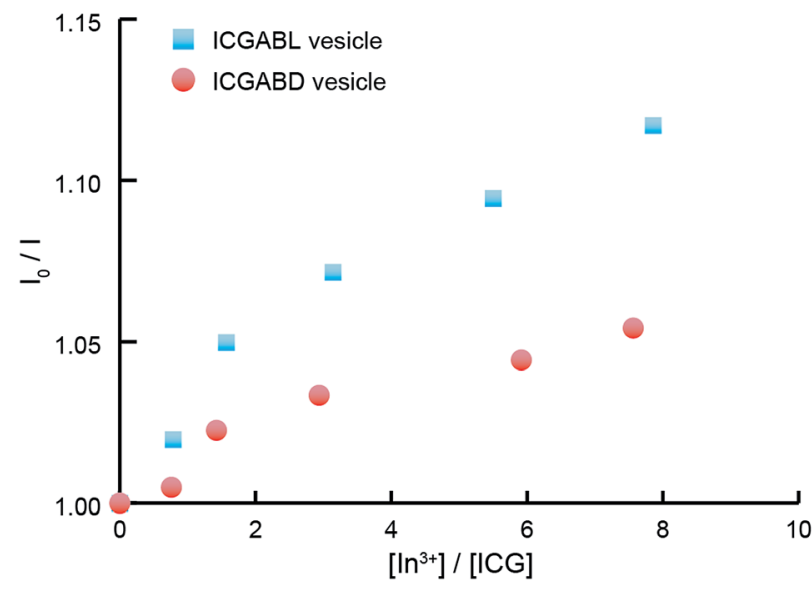

Fig. 6 Stern-Volmer plots of the fluorescence quenching of vesicles containing ICGABL (blue squares) and ICGABD (red circles) by $\ln ^{3+}$ ions.

those containing ICGABD according to the Stern-Volmer plots (Fig. 6). The molecular orientation of ICGABL and ICGABD in each vesicle is therefore controlled to locate at the outside and inside of the vesicles, respectively. In these ternary mixtures, the $\mathbf{A}_{3} \mathbf{B L}$ proportions in all the right-handed helix peptides exceed $96 \%$, and the unsymmetric vesicle formation from these ternary mixtures is consistent with our interpretation of the relationship between unsymmetry and composition.

The in vivo dispositions of vesicles containing ICGABL and ICGABD in tumor-bearing mice were analysed by NIRF imaging (Fig. 7 and S6 of ESI $\dagger$ ). These vesicles were prepared in milliQ water, and the concentration of $\mathrm{NaCl}$ was adjusted to the physiological condition $(0.9 \mathrm{wt} \%$ ) by the addition of $\mathrm{NaCl}$. The hydrodynamic diameters of both vesicles before and after the salt addition were $112.8 \mathrm{~nm}$ (PDI of 0.080) and $109.0 \mathrm{~nm}$ (PDI of 0.083 ), respectively. The integrity of the vesicle with the added $\mathrm{NaCl}$ was also confirmed by cryo-TEM of the unsymmetric vesicle prepared from $\mathbf{A}_{\mathbf{3}} \mathbf{B L}+\mathbf{A B D}$; the membrane maintained its impermeable property against FITC-dextran incorporated into the vesicle upon the change of ionic strength. $^{33}$

The accumulated amounts of the vesicles in tumor in the right femoral region and in the healthy part of the left femoral region were estimated from the fluorescence intensities of the images. Fig. 7 shows that the vesicle containing ICGABD was accumulated more in the tumor than the vesicle containing ICGABL. Furthermore, the NIRF images obtained at 5 min after injection show that the vesicle containing ICGABD spread over the entire body to fluoresce more intensely than the vesicle containing ICGABL (Fig. 7b). The vesicle concentration in the blood stream at $5 \mathrm{~min}$ after injection should be therefore be higher for the vesicle containing ICGABD than for that containing ICGABL. At the moment, the clearance time of the vesicle could not be obtained due to significant capture by the liver; however, these observations support that the vesicle containing ICGABD should remain in the blood stream longer than the vesicle containing ICGABL, which induced the greater accumulation of the former vesicle in tumors compared to the latter. In the case of the vesicle containing ICGABD, the fluorophores were concealed in the unsymmetric vesicle, which should diminish capture by the liver, especially for the ICG fluorophore.

Some nanoparticles have been reported to trigger IgM production when they are dosed to mice. ${ }^{36-38}$ For example, polymeric micelles prepared from poly(Sar) ${ }_{64}-b$-poly(L-lactic acid $)_{30}$ induced the production of anti-poly(sarcosine) IgM and IgG with a long memory. The amounts of antipoly(sarcosine) IgM produced by the current vesicles were analysed by ELISA using Lactosome as the positive control (Fig. 8). The vesicles containing ICG-peptides triggered the production of IgM but in less amounts compared with Lactosome. Ishida et al. pointed out that the surface density influences the amount of IgM production. ${ }^{38}$ On the other hand, we have reported that the high surface density of the poly(sarcosine) chains of nanoparticles suppresses IgM production. $^{39}$ In the present case, the $\mathrm{A}_{3} \mathrm{~B}$-type peptide extruded the bulky $\mathrm{A}_{3}$ moieties outside, and the helical blocks were tightly packed due to stereo-complex formation. The surface density of poly(sarcosine) should therefore be higher with the current vesicles compared to with Lactosome. This higher surface density might explain the ability of the current vesicles to suppress IgM production even though the vesicles have larger diameters (about $100 \mathrm{~nm}$ ) compared to Lactosome (35 $\mathrm{nm})$.

The vesicle containing ICGABL triggered IgM production more than the vesicle containing ICGABL. Since the ICG moieties of the vesicle containing ICGABL are exposed to the outside of the vesicle, the surface may have more defects than the vesicle containing ICGABD, making recognition by B cells easier. It is considered that the unsymmetric vesicles also have different stimulation properties to the immune system. 




Fig. 7 Accumulations of the vesicles containing ICGABL and ICGABD in tumor-bearing mice by NIRF imaging $(n=3)$. NIRF images were taken at 15 min, $1 \mathrm{~h}, 3 \mathrm{~h}, 6 \mathrm{~h}, 9 \mathrm{~h}$ and $24 \mathrm{~h}$ after administration. (a) The vertical axis represents ROI at tumor and background. The $p$-value: * $p<0.05$, ** $p<$ 0.01 (time point is $24 \mathrm{~h}$ ). (b) In vivo NIRF images taken at $24 \mathrm{~h}$ after administration. A Clairvivo OPT system was used for the NIRF imaging.

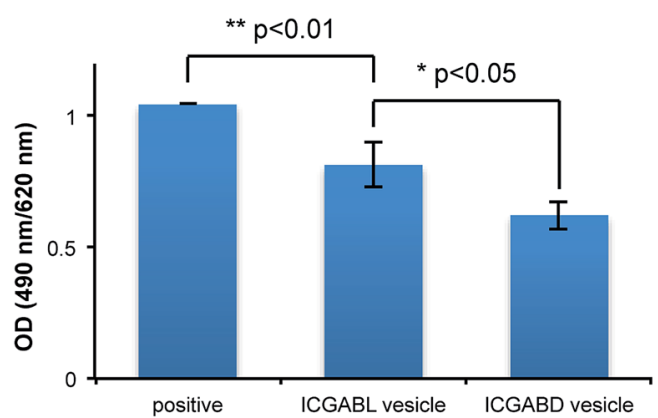

Fig. 8 The production of anti-poly(sarcosine) IgM evaluated by ELISA using a Lactosome plate. The serum was taken at seven days after the dose of Lactosome (positive), the vesicles containing ICGABL (ICGABL vesicle), or the vesicles containing ICGABD (ICGABD vesicle). The $p$-value: $* p<0.05, * * p 0.01$.

\section{Conclusions}

Unsymmetric vesicles were prepared from mixtures of $\mathrm{A}_{3} \mathrm{~B}$-type right-handed helices and $\mathrm{AB}$-type left-handed helices in milliQ water utilising stereo-complex formation, dipole-dipole interactions and steric effects. More than $60 \% \mathrm{~A}_{3} \mathrm{~B}$-type peptides in all the right-handed helices is necessary to obtain unsymmetric vesicles as a result of steric effects. In other words, the vesicles can possibly contain up to $40 \%$ functionalised AB-type righthanded helix peptide in all the right-handed helices where all the functional groups are exposed to the outside of the vesicle. Furthermore, the functional groups can be concealed in the inner surface of the vesicle by using a functionalised AB-type left-handed helix peptide. As shown in the present paper, the vesicles show different behaviours regarding in vivo disposition and reaction with the immune system depending on the locations of the function groups on the vesicle. We are currently trying to increase the vesicle accumulation amount in tumors and develop a bimodal imaging vesicle with the unsymmetric vesicle.

\section{Acknowledgements}

This study is a part of the Innovative Techno-Hub for Integrated Medical Bio-Imaging of the Project for Developing Innovation Systems from the Ministry of Education, Culture, Sports, Science and Technology (MEXT), Japan.

\section{Notes and references}

1 A. D. Bangham and R. W. Horne, J. Mol. Biol., 1964, 8, 660668.

2 B. M. Discher, Y.-Y. Won, D. S. Ege, J. C.-M. Lee, F. S. Bates, D. E. Discher and D. A. Hammer, Science, 1999, 284, 11431146.

3 D. E. Discher and A. Eisenberg, Science, 2002, 297, 967-973. 4 T. Koga, K. Taguchi, Y. Kobuke, T. Kinoshita and M. Higuchi, Chem.-Eur. J., 2003, 9, 1146-1156.

5 Z. Liu, J. Qiao, Y. Tian, M. Wu, Z. Niu and Y. Huang, Langmuir, 2014, 30, 8938-8944.

6 J. N. Israelachvili, D. J. Mitchell and B. W. Ninham, J. Chem. Soc., Faraday Trans. 2, 1976, 72, 1525-1568.

7 J. C. M. van Hest, D. a. P. Delnoye, M. W. P. L. Baars, M. H. P. van Genderen and E. W. Meijer, Science, 1995, 268, 1592-1595.

8 Z. Ge and S. Liu, Macromol. Rapid Commun., 2009, 30, 15231532.

9 E. Braun, Y. Eichen, U. Sivan and G. Ben-Yoseph, Nature, 1998, 391, 775-778. 
10 T. Scheibel, R. Parthasarathy, G. Sawicki, X.-M. Lin, H. Jaeger and S. L. Lindquist, Proc. Natl. Acad. Sci. U. S. A., 2003, 100, 4527-4532.

11 P. Broz, S. Driamov, J. Ziegler, N. Ben-Haim, S. Marsch, W. Meier and P. Hunziker, Nano Lett., 2006, 6, 2349-2353.

12 A. Blanazs, S. P. Armes and A. J. Ryan, Macromol. Rapid Commun., 2009, 30, 267-277.

13 A. Dehsorkhi, V. Castelletto, I. W. Hamley, J. Seitsonen and J. Ruokolainen, Langmuir, 2013, 29, 14246-14253.

14 X. J. Loh, J. del Barrio, T.-C. Lee and O. A. Scherman, Chem. Commun., 2014, 50, 3033-3035.

15 P. P. Ghoroghchian, P. R. Frail, K. Susumu, D. Blessington, A. K. Brannan, F. S. Bates, B. Chance, D. A. Hammer and M. J. Therien, Proc. Natl. Acad. Sci. U. S. A., 2005, 102, 2922-2927.

16 R. Weissleder, Science, 2006, 312, 1168-1171.

17 K. Cho, X. Wang, S. Nie, Z. Chen (Georgia) and D. M. Shin, Clin. Cancer Res., 2008, 14, 1310-1316.

18 H. Tanisaka, S. Kizaka-Kondoh, A. Makino, S. Tanaka, M. Hiraoka and S. Kimura, Bioconjugate Chem., 2008, 19, 109-117.

19 Q. Liu, H. Zhu, J. Qin, H. Dong and J. Du, Biomacromolecules, 2014, 15, 1586-1592.

20 F. Ye, Å. Barrefelt, H. Asem, M. Abedi-Valugerdi, I. El-Serafi, M. Saghafian, K. Abu-Salah, S. Alrokayan, M. Muhammed and M. Hassan, Biomaterials, 2014, 35, 3885-3894.

21 Y. Matsumura and H. Maeda, Cancer Res., 1986, 46, 63876392.

22 A. Makino, S. Kizaka-Kondoh, R. Yamahara, I. Hara, T. Kanzaki, E. Ozeki, M. Hiraoka and S. Kimura, Biomaterials, 2009, 30, 5156-5160.

23 H. Cabral, Y. Matsumoto, K. Mizuno, Q. Chen, M. Murakami, M. Kimura, Y. Terada, M. R. Kano, K. Miyazono, M. Uesaka, N. Nishiyama and K. Kataoka, Nat. Nanotechnol., 2011, 6, 815-823.
24 K. Maruyama, Adv. Drug Delivery Rev., 2011, 63, 161-169.

25 J. C. Kraft, J. P. Freeling, Z. Wang and R. J. Y. Ho, J. Pharm. Sci., 2014, 103, 29-52.

26 Y. J. Jun, M. K. Park, V. B. Jadhav, J. H. Song, S. W. Chae, H. J. Lee, K. S. Park, B. Jeong, J. H. Choy and Y. S. Sohn, J. Controlled Release, 2010, 142, 132-137.

27 M.-H. Lai, S. Lee, C. E. Smith, K. Kim and H. Kong, ACS Appl. Mater. Interfaces, 2014, 6, 10821-10829.

28 D. Kokuryo, Y. Anraku, A. Kishimura, S. Tanaka, M. R. Kano, J. Kershaw, N. Nishiyama, T. Saga, I. Aoki and K. Kataoka, J. Controlled Release, 2013, 169, 220-227.

29 J.-H. Fuhrhop and J. Mathieu,J. Chem. Soc., Chem. Commun., 1983, 144-145.

30 J. H. Fuhrhop and D. Fritsch, Acc. Chem. Res., 1986, 19, 130137.

31 Z. Xiao, M. Xu, M. Li, Z. Lu and Y. Wei, Supramol. Sci., 1998, 5, 619-622.

32 S. Pautot, B. J. Frisken and D. A. Weitz, Proc. Natl. Acad. Sci. U. S. A., 2003, 100, 10718-10721.

33 A. Uesaka, M. Ueda, T. Imai, J. Sugiyama and S. Kimura, Langmuir, 2014, 30, 4273-4279.

34 M. Ueda, A. Makino, T. Imai, J. Sugiyama and S. Kimura, Soft Matter, 2011, 7, 4143-4146.

35 M. Ueda, A. Makino, T. Imai, J. Sugiyama and S. Kimura, Chem. Commun., 2011, 47, 3204-3206.

36 E. Hara, A. Makino, K. Kurihara, F. Yamamoto, E. Ozeki and S. Kimura, Int. Immunopharmacol., 2012, 14, 261-266.

37 E. Hara, A. Makino, K. Kurihara, M. Sugai, A. Shimizu, I. Hara, E. Ozeki and S. Kimura, Biochim. Biophys. Acta, Gen. Subj., 2013, 1830, 4046-4052.

38 T. Ishida, M. Harada, X. Y. Wang, M. Ichihara, K. Irimura and H. Kiwada, J. Controlled Release, 2005, 105, 305-317.

39 E. Hara, M. Ueda, C. J. Kim, A. Makino, I. Hara, E. Ozeki and S. Kimura, J. Pept. Sci., 2014, 20, 570-577. 